\title{
DISCOVERY OF X-RAY PULSATION FROM THE GEMINGA-LIKE PULSAR PSR J2021+4026
}

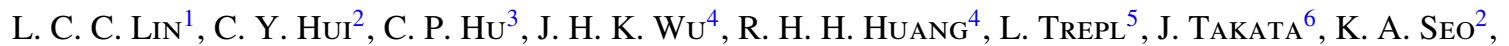

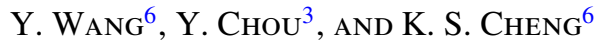 \\ ${ }^{1}$ General Education Center, China Medical University, Taichung 40402, Taiwan \\ ${ }^{2}$ Department of Astronomy and Space Science, Chungnam National University, Daejeon, Korea; cyhui@cnu.ac.kr \\ ${ }^{3}$ Graduate Institute of Astronomy, National Central University, Jhongli 32001, Taiwan \\ ${ }^{4}$ Institute of Astronomy, National Tsing-Hua University, Hsinchu 30013, Taiwan \\ ${ }^{5}$ Astrophysikalisches Institut und Universitäts-Sternwarte, Universität Jena, Schillergäßchen 2-3, D-07745 Jena, Germany \\ ${ }^{6}$ Department of Physics, University of Hong Kong, Pokfulam Road, Hong Kong, China \\ Received 2013 March 4; accepted 2013 May 3; published 2013 May 24
}

\begin{abstract}
We report the discovery of an X-ray periodicity of $\sim 265.3 \mathrm{~ms}$ from a deep XMM-Newton observation of the radioquiet $\gamma$-ray pulsar, PSR J2021+4026, located at the edge of the supernova remnant G78.2+2.1 ( $\gamma$-Cygni). The detected frequency is consistent with the $\gamma$-ray pulsation determined by the observation of the Fermi Gamma-ray Space Telescope at the same epoch. The X-ray pulse profile resembles the modulation of a hot spot on the surface of the neutron star. The phase-averaged spectral analysis also suggests that the majority of the observed X-rays have thermal origins. This is the third member in the class of radio-quiet pulsars with significant pulsations detected from both X-ray and $\gamma$-ray regimes.
\end{abstract}

Key words: gamma rays: stars - ISM: individual objects (G78.2+2.1) - pulsars: individual (PSR J2021+4026) X-rays: stars

Online-only material: color figure

\section{INTRODUCTION}

Before the launch of the Fermi Gamma-ray Space Telescope, only one radio-quiet $\gamma$-ray pulsar was known, namely Geminga (i.e., PSR B0633+17; Bertsch et al. 1992). The high sensitivity of the Large Area Telescope (LAT) on board Fermi enabled an efficient search of $\gamma$-ray pulsars (Abdo et al. 2008, 2010a; Ackermann et al. 2011). Soon after the commencement of the mission, Abdo et al. (2009a) reported the detections of 16 pulsars, 13 of which were determined to be radio-quiet after the dedicated radio pulsation search (Camilo et al. 2009; Abdo et al. $2010 \mathrm{~b}$ ). The population of radio-quiet $\gamma$-ray pulsars has been expanded to 22 based on the extensive searches of Saz Parkinson et al. (2010). Due to the improved search techniques provided by Kerr (2011) and Pletsch (2011), in total there are 31 currently known radio-quiet $\gamma$-ray pulsars (Pletsch et al. 2012a, 2012b).

For this class of pulsars, the lack of knowledge in the phase relationship between the $\gamma$-ray light curve and that in radio leads to an ambiguity in investigating their emission region and physical processes (see the discussion in Trepl et al. 2010). Therefore, pulsation searches in other frequencies are fundamentally important for a better understanding of the physics of this class of pulsars. Thus far, X-ray pulsations have only been detected for Geminga (Halpern \& Holt 1992) and PSR J0007+7303 (Lin et al. 2010; Caraveo et al. 2010). In this work, we report our results of an X-ray periodicity search for another member in this class-PSR J2021+4026.

PSR J2021+4026 is one of the brightest $\gamma$-ray pulsars, which is associated with the supernova remnant (SNR) G78.2+2.1, and was detected by Fermi LAT shortly after its operation began (Abdo et al. 2009b). Its possible association with the SNR suggests that it is at a distance of $\sim 1.5 \mathrm{kpc}$ (Trepl et al. 2010 and references therein). Its proximity makes it an ideal target for multi-wavelength investigations. Several efforts have been devoted to searching for its radio counterpart; however, no radio pulsar associated with PSR J2021+4026 has been detected so far (Becker et al. 2004; Trepl et al. 2010; Ray et al. 2011).

The timing ephemeris of PSR J2021+4026 has been determined by Fermi LAT at different epochs (Abdo et al. 2009a, 2010a; Ackermann et al. 2011; Ray et al. 2011; Weisskopf et al. 2011) and no obvious glitch was detected between the epoch of MJD 54680 and MJD 55600. Its observed period and period derivative $\left(P=265 \mathrm{~ms}\right.$ and $\left.\dot{P}=5.48 \times 10^{-14} \mathrm{~s} \mathrm{~s}^{-1}\right)$ imply a spin-down age of $\sim 77 \mathrm{kyr}$, a surface dipolar magnetic field strength of $\sim 4 \times 10^{12} \mathrm{G}$, and a spin-down power of $\sim 10^{35} \mathrm{erg} \mathrm{s}^{-1}$. At a distance of $1.5 \mathrm{kpc}$, its $\gamma$-ray conversion efficiency is not dissimilar from that of Geminga (Trepl et al. 2010).

In the X-ray regime, a previously unidentified X-ray source, 2XMM J202131.0+402645, has been identified as a promising counterpart of PSR J2021+4026 (Trepl et al. 2010; Weisskopf et al. 2011). This source is found to be the only non-variable $\mathrm{X}$-ray object without any optical/IR counterpart within its $\gamma$-ray error circle. Its association with the pulsar is reinforced by the fact that its X-ray position is consistent with the optimal $\gamma$-ray timing solution.

In order to firmly establish the link between this X-ray source and PSR J2021+4026, an X-ray periodicity search and a detailed spectroscopy are necessary. Nevertheless, this is forbidden by the limited temporal resolution and the small photon statistics of the archival data. In view of this, we have investigated this Geminga-like pulsar with deep XMM-Newton observations. In this Letter, we report the discovery of the X-ray pulsation from PSR J2021+4026. This is the third member of this class with significant pulsation detected from both the X-ray and $\gamma$-ray regimes.

\section{OBSERVATIONS AND DATA ANALYSIS}

We observed PSR J2021+4026 with XMM-Newton on 2012 April 11 for a total exposure of $\sim 133$ ks (Obs. ID: 0670590101; 
PI: Hui). The median satellite boresight pointing during this observation is R.A. $=20^{\mathrm{h}} 21^{\mathrm{m}} 30^{\mathrm{s}} .56$ decl. $=+40^{\circ} 26^{\prime} 46^{\prime \prime} .8(\mathrm{~J} 2000)$, which is the position of 2XMM J202131.0+402645 determined by Trepl et al. (2010). While the MOS1/2 CCDs were operated in the full-window mode, PN CCD was operated in the small-window mode with a temporal resolution of $\sim 5.7 \mathrm{~ms}$, which enables the pulsation search for the first time. With the most updated instrumental calibration, we generated the event lists from the raw data obtained from all EPIC instruments with the tasks emproc and epproc of the XMM-Newton Science Analysis Software (XMMSAS, version 12.0.1). We selected only those events for which the pattern was between 0 and 12 for MOS cameras and 0 and 4 for the PN camera. We also noted that our data have been contaminated by the hard X-ray background flare. After removing all events which are potentially contaminated, the effective exposures are found to be 85,72 , and $77 \mathrm{ks}$ for MOS1, MOS2, PN, respectively.

\subsection{Timing Analysis}

For timing analysis, we solely utilized the PN data for the pulsation search. To determine the source position of 2XMM J202131.0+402645, we ran the source detection with the XMMSAS task edetect_chain. The source can be significantly detected with the signal-to-noise ratio of $\sim 72 \sigma$ at (J2000) R.A. $=20^{\mathrm{h}} 21^{\mathrm{m}} 30^{\mathrm{s}} .53$, decl. $=+40^{\circ} 26^{\prime} 45^{\prime \prime} .5$ with an uncertainty of $\sim 0$.'4. We extracted the events within a circular region of a $20^{\prime \prime}$ radius centered at this position, which corresponds to an encircle energy function of $\sim 76 \%$. Within an energy band of $0.15-12 \mathrm{keV}, 3174$ counts were available for the timing analysis. With the aid of the task barycen, the arrival times of all the selected events were barycentric-corrected with the aforementioned X-ray position and the latest JPL DE405 earth ephemeris.

Our search around the rotational frequency of PSR $\mathrm{J} 2021+4026$ with the method of epoch-folding results in a very significant detection of X-ray pulsation from $2 \mathrm{XMM}$ $\mathrm{J} 202131.0+402645$. Using either the Rayleigh test (Mardia 1972; Gibson et al. 1982) or the $H$-test (de Jager et al. 1989; de Jager \& Büsching 2010), a significant peak is found at a frequency of $3.7689937(9) \mathrm{Hz}(=0.26532281(6) \mathrm{s})$ with $Z_{1}^{2}=126$ and $H=133$, respectively. This corresponds to the random probability of $<10^{-14}$ (de Jager \& Büsching 2010). The quoted uncertainty of our detected frequency is determined by following the method used in Leahy (1987).

Although the ephemeris of PSR J2021+4026 has been determined several times by Fermi LAT observations at different epochs (Abdo et al. 2009a, 2010a; Ackermann et al. 2011; Ray et al. 2011; Weisskopf et al. 2011), the epoch of our XMMNewton observation (i.e., MJD 56028) has not been covered. In order to avoid the uncertainties introduced by extrapolating these timing models to our observation epoch, we derived a $\gamma$-ray ephemeris which is contemporaneous with this X-ray observation for comparing the temporal properties in both regimes.

We obtained the Fermi LAT data in the energy range of $0.1-300 \mathrm{GeV}$ within a circular region of interest with a 0.8 radius from the X-ray position of PSR J2021+4026. In order to achieve a reasonable signal-to-noise ratio and with the influence of the accumulated timing noise minimized, we adopted a data span of $\sim 1$ yr, MJD 55900-56280, which brackets the epoch the XMMNewton observation. For data reduction, the Fermi Science Tools v9r23p1 package, available from the Fermi Science Support
Table 1

Local Ephemeris of PSR J2021+4026 Derived from LAT Data Which Brackets the XMM-Newton Observation on MJD 56028

\begin{tabular}{lc}
\hline \hline Pulsar name & PSR J2021+4026 \\
Valid MJD range & $55908-56274$ \\
R.A., $\alpha$ & $20: 21: 30.733$ \\
Decl., $\delta$ & $+40: 26: 46.04$ \\
Pulse frequency, $f\left(\mathrm{~s}^{-1}\right)$ & $3.768995206(2)$ \\
First derivative of pulse frequency, $\dot{f}\left(\mathrm{~s}^{-2}\right)$ & $-8.166(3) \times 10^{-13}$ \\
Second derivative of pulse frequency, $\ddot{f}\left(\mathrm{~s}^{-3}\right)$ & $-2(1) \times 10^{-22}$ \\
Third derivative of pulse frequency, $\ddot{f}\left(\mathrm{~s}^{-4}\right)$ & $1(2) \times 10^{-29}$ \\
Epoch of frequency determination $(\mathrm{MJD})$ & 56000 \\
Solar system ephemeris model & $\mathrm{DE} 405$ \\
Time system & TDB
\end{tabular}

Note. The numbers in parentheses denote errors in the last digit.

Center, ${ }^{7}$ was used. We used Pass 7 data and selected events in the "Source" class (i.e., event class 2) only. In addition, we excluded the events with zenith angles larger than $100^{\circ}$ to greatly reduce the contamination by Earth albedo $\gamma$-rays. To determine the pulse times of arrivals (TOAs), we first constructed a template based on the timing model reported by Ray et al. (2011) with the method of Gaussian kernel density estimation. By crosscorrelating the template with unbinned geocentered data, with each photon assigned a phase in accordance with the model reported by Ray et al. (2011), we measured the TOAs from 25 segments of adopted data span.

Using TEMPO2, we fitted the TOAs to a timing model including $f, \dot{f}, \ddot{f}$, and $\dddot{f}$. All spin parameters are allowed to be free with the high-order derivatives accounting for most of the red noise. For the position, as the short data span does not allow a fit, we fixed it at the X-ray position determined by Chandra throughout the analysis (Weisskopf et al. 2011). The results are summarized in Table 1. Since the high-order frequency derivatives are dominated by the timing noise, we stress that their values do not reflect the intrinsic rotational properties of the pulsar. Using the parameters in Table 1, the extrapolated $f$ and $\dot{f}$ are consistent with the ephemeris at the specified epochs reported by earlier works within the statistical uncertainties. According to this local ephemeris, the spin frequency of PSR J2021+4026 at the start of the good time interval (MJD 56028.31153) of our XMM-Newton observation is $3.768993208(2) \mathrm{Hz}$, which is consistent with the detected X-ray pulsation (i.e., $f=3.7689937(9) \mathrm{Hz}$ ).

To compare the X-ray and $\gamma$-ray pulse profiles, the photons extracted from our XMM-Newton observation and those obtained from Fermi within MJD 55910-56110 were all folded in accordance with our derived $\gamma$-ray ephemeris (i.e., Table 1) with epoch zero at MJD 56028. For further examination of the properties of the X-ray pulsation, we performed the energyresolved timing analysis by dividing the $\mathrm{X}$-ray data into three consecutive bands: $0.15-0.7 \mathrm{keV}$ (soft), $0.7-2 \mathrm{keV}$ (medium), and 2-12 keV (hard). The phase-aligned pulse profiles at different energy ranges are shown in Figure 1. Apparently, the X-ray pulsation is most significant in the medium band $\left(Z_{1}^{2}=166\right)$. In the hard band, there is a very marginal pulse detection of $Z_{1}^{2}=17.8$. However, this corresponds to a much higher random probability of $\sim 8 \times 10^{-4}$. In the soft band, there is no conclusive evidence for the pulsation.

There is a recognizable phase offset between the $\gamma$-ray and the X-ray profiles (see the left panel in Figure 2). To quantify

\footnotetext{
http://fermi.gsfc.nasa.gov/ssc/data/analysis/software/
} 


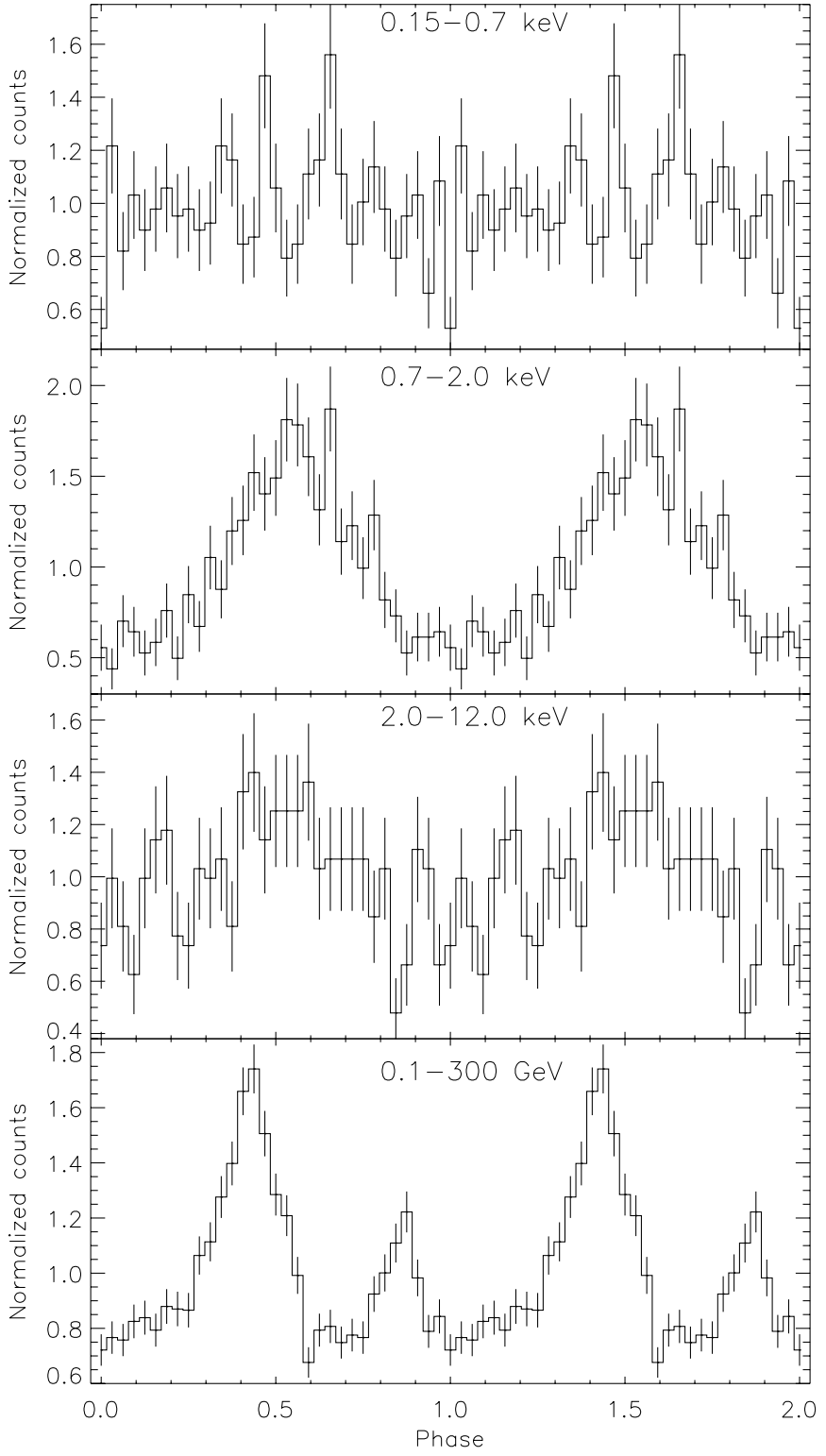

Figure 1. Folded light curves of PSR J2021+4026 in different energy ranges. Each panel shows the pulse profile of 32 bins in the energy band specified in the legend. All the light curves were folded with the timing parameters at the epoch/phase zero of MJD 56028 derived from Table 1. the phase offset, we computed the cross-correlation between these two phase-aligned light curves. The result is shown in the right panel of Figure 2. The cross-correlation coefficient attains the maximum value at a phase lag of -0.138 . In order to estimate the uncertainty of cross-correlation, we used Monte Carlo simulation to obtain the distribution of phase lag. The simulated X-ray light curves were generated by the original one plus a random series of variates sampled from a Gaussian distribution with a mean of 0 and a standard derivation of $1 \sigma$ corresponding to the errors of observed folded light curve. With 10,000 trials, the standard deviation is found to be 0.024 , which suggests that the aforementioned phase lag is detected at a confidence level of $>5 \sigma$.

\subsection{Spectral Analysis}

Besides detecting the X-ray pulsation, our deep XMM-Newton observation is also able to place a tight constraint on the spectral properties of PSR J2021+4026. For spectral analysis, we utilized the data obtained from all three EPIC cameras. With the aid of the XMMSAS task epatplot, all the EPIC data are found to be unaffected by CCD pileup. We extracted the spectrum of PSR J2021+4026 from circles with a radius of $20^{\prime \prime}$ centered at its nominal X-ray position (see Section 2.1) in MOS1, MOS2, and PN cameras, respectively. The background spectra were extracted from the nearby regions in the corresponding CCDs, which are source free and with sufficient sizes to enable a less biased sampling. The response files were generated by the XMMSAS tasks rmfgen and arfgen. After background subtraction, we have 1159 counts collected by EPIC in the energy range of $0.5-10 \mathrm{keV}$. The photon statistic is $\sim 4$ times higher than that used in the spectral analysis reported by Weisskopf et al. (2011). We grouped each spectrum dynamically so as to achieve the same signal-to-noise ratio in each data set. In order to tightly constrain the spectral parameters, we fitted the data obtained from three cameras simultaneously with XSPEC 12.6.0. For a conservative estimation of uncertainties, the quoted errors of the spectral parameters are $1 \sigma$ for two parameters of interest (i.e., $\Delta \chi^{2}=2.30$ above the minimum) for the single-component models and $1 \sigma$ for four parameters of interest (i.e., $\Delta \chi^{2}=4.72$ above the minimum) for the multicomponent models.

With the tested single-component models, we found that the pulsar spectrum cannot be appropriately described by these simple scenarios. The absorbed blackbody results in a relatively
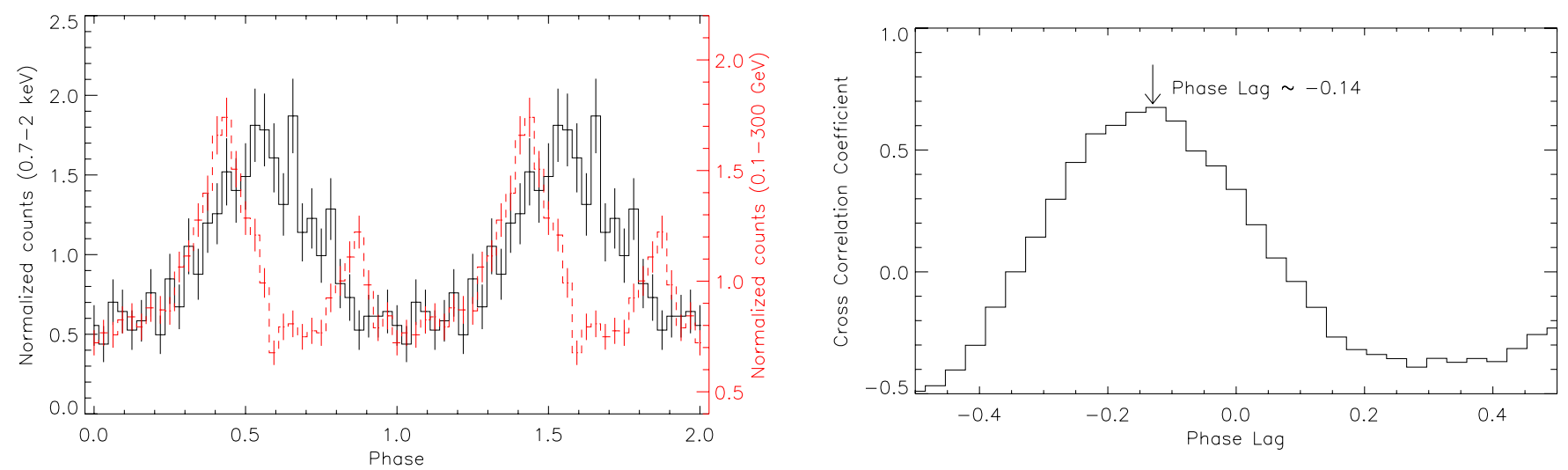

Figure 2. Correlation of the X-ray and $\gamma$-ray pulsations. Left panel: the light curves of PSR J2021+4026 in the X-ray and $\gamma$-ray bands both folded at epoch zero of MJD 56028 with 32 bins using the same ephemeris reported in this Letter. The pulse profiles in $0.7-2 \mathrm{keV}$ and $0.1-300 \mathrm{GeV}$ are shown by solid and dashed lines, respectively. Right panel: cross-correlation of the X-ray and $\gamma$-ray pulse profiles is shown. The coefficient has a maximum value at a phase lag of -0.14 .

(A color version of this figure is available in the online journal.) 


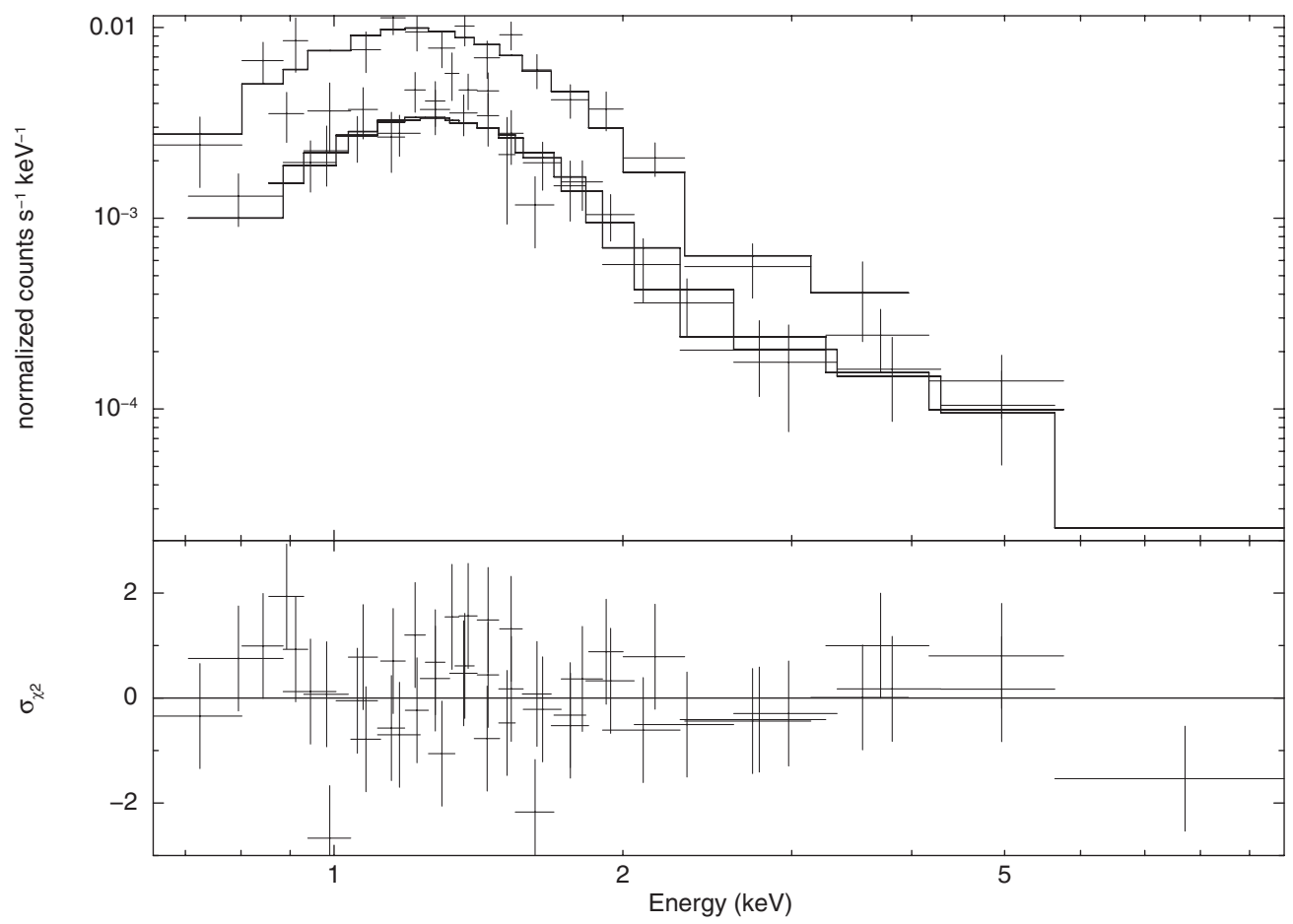

Figure 3. Phase-averaged energy spectrum of PSR J2021+4026 in 0.5-10 keV. The X-ray emission from the position of PSR J2021+4026 as observed with the PN (upper spectrum) and MOS1/2 detectors (lower spectra) is simultaneously fitted to an absorbed blackbody plus power-law model (upper panel) and contributions to the $\chi^{2}$ fit statistic (lower panel).

poor goodness of fit $\left(\chi^{2}=65.33\right.$ for 46 degrees of freedom (dof)). On the other hand, although the absorbed power-law model results in an acceptable goodness of fit $\left(\chi^{2}=52.78\right.$ for 46 dof $)$, it yields a very large photon index $\left(\Gamma=4.4_{-0.5}^{+0.6}\right)$ which is far steeper than that expected for a pulsar (cf. Cheng \& Zhang 1999). In both of these single-component fits, systematic deviations have been noted in the fitting residuals for energies larger than $\sim 3 \mathrm{keV}$. All these suggest that the X-ray emission of PSR J2021+4026 might consist of more than one spectral component.

We proceeded to examine the spectrum with multi-component models. We found that the blackbody plus power-law model $(\mathrm{BB}+\mathrm{PL})$ can describe the data reasonably well $\left(\chi^{2}=41.55\right.$ for 44 dof). It yields a column density of $n_{\mathrm{H}}=\left(7.0_{-2.7}^{+4.1}\right) \times$ $10^{21} \mathrm{~cm}^{-2}$, a temperature of $k T=0.24 \pm 0.06 \mathrm{keV}$, a blackbody emitting region with a radius of $R=251_{-132}^{+537} d_{1.5} \mathrm{~m}$, a photon index of $\Gamma=1.2_{-1.2}^{+1.7}$, and a normalization for the power-law component at $1 \mathrm{keV}$ of $\left(2.8_{-2.6}^{+14.1}\right) \times 10^{-6}$ photons $\mathrm{keV}^{-1} \mathrm{~cm}^{-2} \mathrm{~s}^{-1}$, where $d_{1.5}$ represents the distance to PSR J2021+4026 in unit of $1.5 \mathrm{kpc}$. The best-fit BB+PL model and the residuals for the X-ray spectrum of PSR J2021+4026 are shown in Figure 3. On the other hand, a pure thermal scenario consisting of two blackbody components $(\mathrm{BB}+\mathrm{BB})$ also results in a comparable goodness of fit $\left(\chi^{2}=40.19\right.$ for $44 \mathrm{dof}$ ). It yields a set of best-fit parameters of $n_{\mathrm{H}}=$ $\left(6.7_{-2.5}^{+3.6}\right) \times 10^{21} \mathrm{~cm}^{-2}, k T_{1}=1.4_{-0.6}^{+1.8} \mathrm{keV}, R_{1}=3.6_{-2.5}^{+6.4} d_{1.5} \mathrm{~m}$, $k T_{2}=0.25 \pm 0.05 \mathrm{keV}$, and $R_{2}=223_{-106}^{+320} d_{1.5} \mathrm{~m}$. Both of these composite models imply an unabsorbed flux at the level of $\sim 10^{-13} \mathrm{erg} \mathrm{cm}^{-2} \mathrm{~s}^{-1}$.

\section{DISCUSSION}

In this Letter, we report our detection of an X-ray pulsation from 2XMM J202131.0+402645. The detected spin frequency is consistent with the $\gamma$-ray pulsation of PSR J2021+4026 at the same epoch. This provides clear evidence that the observed $\mathrm{X}$-rays are indeed from PSR J2021+4026.

The X-ray pulse profile in $0.7-2 \mathrm{keV}$ resembles the modulation that resulted from a rotating neutron star while a hot spot on the stellar surface sweeps across our line of sight (e.g., Hui $\&$ Cheng 2004; Pechenick et al. 1983). This is consistent with the scenario inferred from the phase-averaged spectral analysis which favors a model of a blackbody plus power law or a composite blackbody model. In both of these best-fit models, a blackbody component with a temperature of $k T \sim 0.25 \mathrm{keV}$ is required. At a distance of $1.5 \mathrm{kpc}$, the best-fit blackbody radius is found to be $\sim 0.2-0.3 \mathrm{~km}$. This is comparable with the canonical size of a polar cap, $R \sqrt{R \omega / c} \sim 280 \mathrm{~m}$, where $R \sim 10 \mathrm{~km}$ represents the typical radius of a neutron star and $\omega$ is the angular frequency of PSR J2021+4026. According to the outer gap model (Takata et al. 2012; Cheng \& Zhang 1999), the temperature of the polar cap heated by the return current injected by the gap is $k T \sim 0.3 \mathrm{keV}$, which is also consistent with the observed value. Furthermore, this spectral component contributes $>80 \%$ to the observed flux in both $\mathrm{BB}+\mathrm{PL}$ and $\mathrm{BB}+\mathrm{BB}$ fits in $0.7-2 \mathrm{keV}$. All these results point to the thermal nature of the observed X-ray pulsation.

In the $\mathrm{BB}+\mathrm{PL}$ fit, the non-thermal component with a photon index of $\Gamma \sim 1.2$ is typical for a canonical pulsar. One possible origin of these non-thermal X-rays is the synchrotron radiation of the relativistic $e^{-} / e^{+}$from the outer magnetospheric gap (Cheng \& Zhang 1999; Takata et al. 2006, 2008), which should give rise to a pulsed signal. Although the best-fit power-law component contributes $>90 \%$ to the observed flux at the energies $>2 \mathrm{keV}$, the evidence for the pulsation in the hard band is marginal. This suggests that the power-law component might be non-pulsed in nature, which can possibly originate from a pulsar wind nebula. Indeed, a putative bow shock associated with PSR J2021+4026 has been marginally resolved 
by a Chandra observation (C. Y. Hui et al. 2013, in preparation; Weisskopf et al. 2011). It might contribute a steady, unpulsed, non-thermal X-ray emission as a DC level which can be found in all rotational phases.

On the other hand, the $\mathrm{BB}+\mathrm{BB}$ model is also able to fit the observed data with a comparable goodness of fit. While the low-temperature component is consistent with that inferred in the $\mathrm{BB}+\mathrm{PL}$ fit, it also requires a hotter component with a much smaller emitting area with a radius of a few meters. This might be a phenomenological two-step adaptation for a wider temperature distribution of the hot polar cap with the hotter component describing the peak of the distribution. The marginal detection of X-ray pulsation in the hard band may have originated from the modulation of this component. However, the inferred temperature, $\sim 2 \times 10^{7} \mathrm{~K}$, is higher than that expected from the heating by the back flow current from the outer gap (Cheng \& Zhang 1999) unless the surface multipolar magnetic field is strong enough to reduce the pure dipolar cap area.

Comparing the phase-aligned X-ray and $\gamma$-ray light curves (Figure 1), their pulse morphologies are clearly different where the $\gamma$-ray profile is narrower and contains at least two peaks. In cross-correlating these two profiles, the cross-correlation coefficient is found to attain a maximum value of $\sim 0.67$ at a phase lag of $\sim-0.14$ as shown in the right panel of Figure 2 . This clearly indicates that the observed X-rays and $\gamma$-rays originated from different sites. By comparing these observed results with the model predictions, the pulsar emission geometry (e.g., magnetic inclination angle, viewing angle) can be constrained. In a previous study, Trepl et al. (2010) modeled the $\gamma$-ray pulse profile of PSR J2021+4026 in the context of the outer gap model. Nevertheless, due to a lack of pulses detected in radio and any other wavelength at that time, there was an uncertainty in determining the leading peak (see Figures 13 and 14 in Trepl et al. 2010). With our detection of an X-ray pulsation, such ambiguity can now be resolved and thus the theoretical investigation of this pulsar can be revisited.

This research has made use of the data obtained through the Fermi Science Support Center Online Service, provided by the NASA/Goddard Space Flight Center. The work is partially supported by the National Science Council through grants NSC
101-2112-M-039-001-MY3. C.Y.H. and K.A.S. are supported by the National Research Foundation of Korea through grant 2011-0023383. C.P.H. and Y.C. are supported by the National Science Council of Taiwan through the grant NSC 101-2112M-008-010. R.H.H.H. acknowledges support from the National Science Council through grants NSC 100-2628-M-007-002MY3 and NSC 100-2923-M-007-001-MY3. J.T. and K.S.C. are supported by a GRF grant of the HK Government under HKU700911P.

Facilities: XMM, Fermi

\section{REFERENCES}

Abdo, A. A., Ackermann, M., Ajello, M., et al. 2009a, Sci, 325, 840 Abdo, A. A., Ackermann, M., Ajello, M., et al. 2009b, ApJS, 183, 46 Abdo, A. A., Ackermann, M., Ajello, M., et al. 2010a, ApJ, 712, 1209 Abdo, A. A., Ackermann, M., Ajello, M., et al. 2010b, ApJ, 711, 64 Abdo, A. A., Ackermann, M., Atwood, W. B., et al. 2008, Sci, 322, 1218 Ackermann, M., Ajello, M., Baldini, L., et al. 2011, ApJ, 726, 35 Becker, W., Weisskopf, M. C., Arzoumanian, Z., et al. 2004, ApJ, 615, 897 Bertsch, D. L., Brazier, K. T. S., Fichtel, C. E., et al. 1992, Natur, 357, 306 Camilo, F., Ray, P. S., Ransom, S. M., et al. 2009, ApJ, 705, 1

Caraveo, P. A., De Luca, A., Marelli, M., et al. 2010, ApJL, 725, L6

Cheng, K. S., \& Zhang, L. 1999, ApJ, 515, 337

de Jager, O. C., \& Büsching, I. 2010, A\&A, 517, L9

de Jager, O. C., Raubenheimer, B. C., \& Swanepoel, J. W. H. 1989, A\&A, 221,180

Gibson, A. I., Harrison, A. B., Kirkman, I. W., et al. 1982, Natur, 296, 833

Halpern, J. P., \& Holt, S. S. 1992, Natur, 357, 222

Hui, C. Y., \& Cheng, K. S. 2004, ApJ, 608, 935

Kerr, M. 2011, ApJ, 732, 38

Leahy, D. A. 1987, A\&A, 180, 275

Lin, L. C. C., Huang, R. H. H., Takata, J., et al. 2010, ApJL, 725, L1

Mardia, K. V. 1972, Statistics of Direction Data (New York: Academic)

Pechenick, K. R., Ftaclas, C., \& Cohen, J. M. 1983, ApJ, 274, 846

Pletsch, H. J. 2011, PhRvD, 83, 122003

Pletsch, H. J., Guillemot, L., Allen, B., et al. 2012a, ApJ, 744, 105

Pletsch, H. J., Guillemot, L., Allen, B., et al. 2012b, ApJL, 755, L20

Ray, P. S., Kerr, M., Parent, D., et al. 2011, ApJS, 194, 17

Saz Parkinson, P. M., Dormody, M., Ziegler, M., et al. 2010, ApJ, 725,571

Takata, J., Chang, H., \& Shibata, S. 2008, MNRAS, 386, 748

Takata, J., Cheng, K. S., \& Taam, R. E. 2012, ApJ, 745, 100

Takata, J., Shibata, S., Hirotani, K., \& Chang, H.-K. 2006, MNRAS, 366, 1310

Trepl, L., Hui, C. Y., Cheng, K. S., et al. 2010, MNRAS, 405, 1339

Weisskopf, M. C., Romani, R. W., Razzano, M., et al. 2011, ApJ, 743, 74 